\title{
Nonlinear phenomena in a high-power heating unit with pulse modulated control
}

\author{
Yuriy Alexsandrovich Gol'tsov, ${ }^{1, *}$, Alexander Stepanovich Kizhuk ${ }^{1}$, and Vasiliy Grigorievich Rubanov ${ }^{1}$ \\ ${ }^{1}$ Department of Engineering Cybernetics, Belgorod State Technological University n.a. V.G. Shukhov, Kostyukov Str., 46, 308012, \\ Belgorod, Russia
}

\begin{abstract}
In this paper we describe the transitions from regular periodic mode to quasiperiodicity that can be observed in a pulse-width modulated control system for a high-power heating unit. The behavior of such a system can be described by a set of two coupled nonautonomous differential equations with discontinuous right-hand sides. We reduce the investigation of this system to the studying of a two-dimensional piecewise-smooth map. We demonstrate how a closed invariant curve associated with quasiperiodic dynamics can arise from a stable periodic motion through a Neimark-Sacker bifurcation. The paper also considers a variety of interesting nonlinear phenomena, including phase-locking modes, multistablilty and hysteretic transitions.
\end{abstract}

\section{Introduction}

The technology of monocrystals growth is a controlled crystallization process, during which the quality of a growing crystal is determined by the accuracy of controlling phase transition conditions $[1,2]$. When growing a sapphire crystal it is necessary to provide the temperature change in the crucible from $25^{\circ} \mathrm{C}$ to $2050^{\circ} \mathrm{C}$ with a certain degree of its increase and decrease.

In this way, it is required an automatic control system with the function of software setting of the temperature variation in the crucible with the required accuracy.

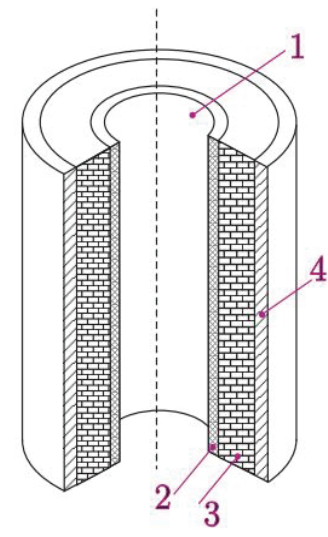

Fig. 1. Schematic diagram of the heating unit.

Figure 1 shows a schematic diagram of the considered heating unit [3]. The heating unit includes the following areas: internal furnace space denoted by number 1, filled with air or gas; nichrome electric heater (denoted by 2), uniformly located in the inner layer of the lining (denoted by number 3 ) which is produced of magnesite bricks. The outer lining layer 4 is constructed of mineral wool that is in a cylindrical beaker made of galvanized steel.

The geometric shape of the furnace is a limited cylinder, with the lining on its top and bottom. The heat transfer in the first two areas is performed only due to thermal conductivity, as the materials of which the furnace lining and the heater are made, are solid and opaque.

In order to perform the synthesis of the control law using a classical approximation method based on the experimental transient behavior of the heating unit we have defined the following transfer function $[3,4]$

$$
W(s) \frac{K}{\left(T_{1} \cdot s+1\right)\left(T_{2} \cdot s+1\right)},
$$

where $K$ is the transfer factor of the plant, and $T_{1}, T_{2}$ are its time constants.

In the modern industry the temperature regulators with thyristor converters are widely used.

However, the use of converters of this type greatly distorts the input current. This causes the appearance of non-sinusoidal modes in the supply network.

In order to eliminate these regimes and to obtain a fast response, an accurate control, and a higher efficiency we have developed a heating unit control system [3, 4] based on a high-frequency switching power electronics converter with pulse-width modulation [5].

At the same time, with real conditions of operation smaller or larger changes of parameters always take place. Such variations often lead to the loss of stability of the normal operation regime and to the appearance of complex dynamical behaviors, including subharmonic, quasiperiodic or chaotic oscillations [6-8].

\footnotetext{
* Corresponding author: umin@mail.ru
} 
The appearance of such oscillatory modes leads not only to the reduction of the control accuracy by several orders, but also to the sudden breakdown of the technological equipment.

The purpose of the present paper is the numerical study of bifurcation phenomena in the pulse modulated control system of a high-power heating unit.

The paper is organized as follows. In sections 2 and 3 we describe a model of the heating unit with pulse-width modulated control. The behavior of such a system may be represented by a two-dimensional piecewise-smooth set of nonautonomous differential equations. We reduce the investigation of this system to studying the dynamics of a two-dimensional piecewise-smooth mapping.

In section 4 , we discuss the bifurcation phenomena occurring in the considered system. The obtained results are summarized in Conclusions.

\section{Continuous - time model}

The dynamics of the heating unit with pulse width modulated control, a continuous linear part which is described by the transfer function (1), can be represented by the following differential equation:

$$
T_{1} T_{2} \frac{d^{2} x}{d t^{2}}+\left(T_{1}+T_{2}\right) \frac{d x}{d t}+x=K U \varphi(\varepsilon)
$$

where $x$ is the temperature in the heating unit, $\varepsilon$ and $\varphi(\varepsilon)$ are the input (error) and output signals of the pulsewidth modulator.

Let us introduce the state variables

$$
x=x_{1}, \frac{d x}{d t}=x_{2} .
$$

Then, the closed-loop system expressed by (2) can now be rewritten as a set of two coupled ordinary differential equations:

$$
\begin{gathered}
\frac{d x_{1}}{d t}=x_{2}, \\
\frac{d x_{2}}{d t}=-\frac{1}{T_{1} T_{2}} x_{1}-\frac{T_{1}+T_{2}}{T_{1} T_{2}} x_{2}+\frac{K U}{T_{1} T_{2}} \varphi(\varepsilon) .
\end{gathered}
$$

The switching function $\varphi(\varepsilon)$ (output signal of the modulator) is defined as:

$$
\begin{gathered}
\varphi(\varepsilon)= \begin{cases}1, & k \cdot T_{0}<t<k \cdot T_{0}+\tau_{k} ; \\
0, & k \cdot T_{0}+\tau_{k}<t<(k+1) \cdot T_{0} ;\end{cases} \\
k=0,1,2, \ldots
\end{gathered}
$$

Here $T_{0}$ is the period of the ramp signal and $\tau_{k}$ is the pulse duration within the time interval $\left.k \cdot T<t<(k+1) \cdot T_{0}\right)$.
In the present analysis we shall consider a system with the proportional feedback corrector and pulsewidth modulation of the first type (see, for example, [6]).

In such a system, depending on the error signal $\varepsilon$ the value of $\tau_{k}$ is determined as:

$$
\tau_{k}=\left\{\begin{array}{cl}
0, & \varepsilon<0 ; \\
T_{0}, & \varepsilon>V_{s} ; \\
\frac{T_{0} \varepsilon}{V_{s}}, & 0 \leq \varepsilon \leq V_{s},
\end{array}\right.
$$

where

$$
\varepsilon=\alpha\left(V_{r e f}-\beta x_{1}\left(k T_{0}\right)\right)
$$

Here $\alpha$ and Vref are the amplification constant and reference signal. $V s$ represents the amplitude of the rump signal and $\beta$ is the sensitivity of the temperature sensor. The mathematical model (3) of such a system may be cast into the form:

$$
\frac{d X}{d t}=\left\{\begin{array}{ccc}
A X+B, & \text { if } & \xi(t, X)>0, \\
A X, & \text { if } & \xi(t, X)<0,
\end{array}\right.
$$

with

$$
X=\left[\begin{array}{l}
x_{1} \\
x_{2}
\end{array}\right], A=\left[\begin{array}{cc}
0 & 1 \\
-\frac{1}{T_{1} T_{2}} & -\frac{T_{2}+T_{2}}{T_{1} T_{2}}
\end{array}\right], B=\left[\begin{array}{c}
0 \\
\frac{K U}{T_{1} T_{2}}
\end{array}\right]
$$

and

$$
\xi(t, X)=V_{r e f}-\beta \cdot x_{1}\left(k \cdot T_{0}\right)-\frac{V_{s}}{\alpha}\left(t / T_{0}-\left\lfloor t / T_{0}\right\rfloor\right) .
$$

Here the number $\left\lfloor t / T_{0}\right\rfloor$ denotes the largest integer not greater than $t / T_{0}$ (i.e., the integer part, or floor, of $\left.t / T_{0}\right)$. The scalar function $\xi(t, X)$ determines the switching manifold

$$
\Sigma=\{(t, X): \xi(t, X)=0\}
$$

that separates the state space into two different regions

$$
\begin{aligned}
& \Sigma_{+}=\{(t, X): \xi(t, X)>0\}, \\
& \Sigma_{-}=\{(t, X): \xi(t, X)<0\}
\end{aligned}
$$

in which the dynamic behavior of the system (5) is governed by different vector fields. It easy to show, that any solution of (5) will intersect the switching manifold (or discontinuity boundary) $\Sigma$ transversely (see [8]). 


\section{Discrete time map}

The investigation of the dynamical system (5) has been reduced to the study of the two-dimensional stroboscopic map.

Within the domain

$$
k T_{0}<t<t_{k}, t_{k}=k T_{0}+\tau_{k},
$$

the system (5) has the form

$$
\frac{d X}{d t}=A X+B, \quad X\left(k \cdot T_{0}\right)=X_{k}
$$

with solution

$$
X(t)=\exp \left(A\left(t-k \cdot T_{0}\right)\left[X_{k}+D\right]-D\right.
$$

At the switching time $t=t_{k}$ :

$$
\begin{gathered}
X\left(t_{k}\right)=\exp \left(A\left(t_{k}-k \cdot T_{0}\right)\left[X_{k}+D\right]-D,\right. \\
D=A^{-1} B .
\end{gathered}
$$

In the subsequent time interval

$$
t_{k}<t<(k+1) T_{0},
$$

system (5) takes the form

$$
\frac{d X}{d t}=A X
$$

with the solution

$$
X(t)=\exp \left(A\left(t-t_{k}\right)\right) X\left(t_{k}\right)
$$

In this way, for $t=(k+1) \cdot T_{0}$

$$
\begin{gathered}
X_{k+1}=\exp \left(A\left((k+1) \cdot T_{0}-t_{k}\right)\right) X\left(t_{k}\right), \\
X_{k+1}=X\left((k+1) \cdot T_{0}\right) .
\end{gathered}
$$

Substituting the expression for $X\left(t_{k}\right)$ into (6), we obtain the two-dimensional piecewise-smooth map [6]

$$
X_{k+1}=\exp \left(A \cdot T_{0}\right)\left[\cdot X_{k}+D\right]-\exp \left(T_{0}-\tau_{k}\right) D
$$

$$
k=0,1,2,3, \ldots
$$

Here $\tau_{k}$

$$
\tau_{k}=\left\{\begin{array}{cl}
0, & \varepsilon_{k}<0 ; \\
T_{0}, & \varepsilon_{k}>V_{s} ; \\
\frac{T_{0} \varepsilon_{k}}{V_{s}}, & 0 \leq \varepsilon_{k} \leq V_{s} ;
\end{array}\right.
$$

with

$$
\varepsilon_{k}=\alpha\left(V_{r e f}-C X_{k}\right)
$$

where $C=(\beta, 0)$ is a row such that $C B=0$.

In the following simulations, we shall use: $T_{1} T_{2}=10240 \mathrm{~s}^{2} ; \quad T 1+T_{2}=352 \mathrm{~s} ; \quad K=327.8 \mathrm{C}^{\circ} / \mathrm{V} ;$ $T_{0}=10 \mathrm{~s} ; U=24 \mathrm{~V} ; \beta=0.01 \mathrm{~V} / \mathrm{C}^{\circ} ; V S=5 \mathrm{~V} ; \alpha>0$; Vref $=5 \mathrm{~V}$.

The gain factor $\alpha$ and the input voltage $U_{0}$ will be used as the main control parameters. The period $T$ of periodic motion is an integer multiple to the rump period $T_{0}$, i.e. $T=m T_{0}, m=1,2, \ldots$ We will refer to this type motion as a period- $m$ cycle or $m$-cycle.

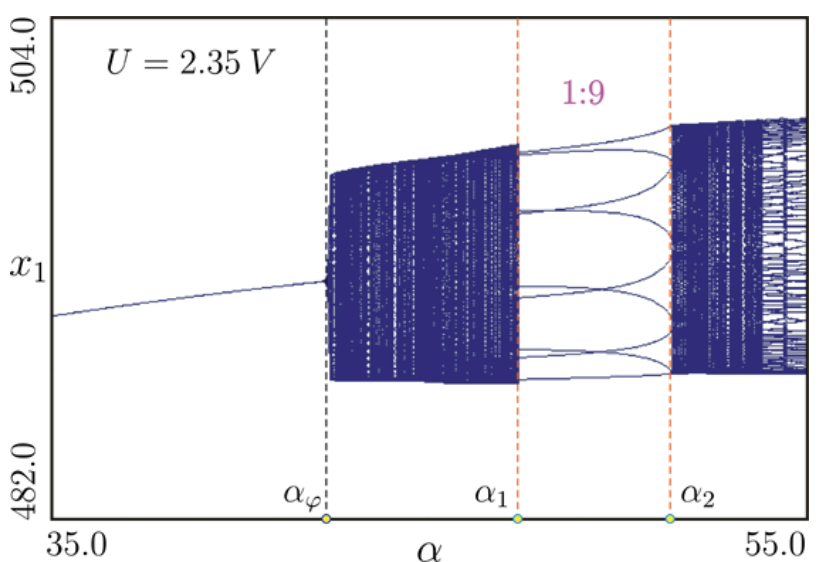

(a)

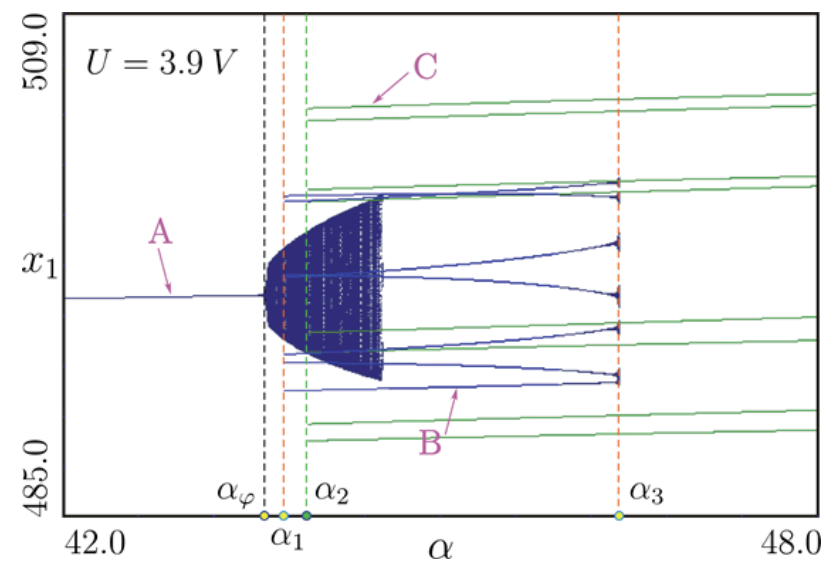

(b)

Fig. 2. (a) Birth of the closed invariant curve associated with quasiperiodic dynamics from the stable 1-cycle through a Neimark-Sacker bifurcation at the point $\alpha_{\varphi}$. (b) Onedimensional bifurcation diagram for $U=3.9 \mathrm{~V}$. The diagram illustrates a form of multistability in which stable 7 - and 8 cycles coexist with a stable closed invariant curve. 


\section{Bifurcation analysis}

Depending on the parameter values, a variety of different bifurcation scenarios is observed.

Figure 2(a) illustrates the appearance of the quasiperiodic orbit form a stable 1 -cycle. For the values of the corrector gain factor $\alpha<\alpha_{\varphi}$ the map (6) shows a single stable $1-$ cycle. As $\alpha$ increases, the $1-$ cycle undergoes a Neimark-Sacker bifurcation at the point $\alpha=\alpha_{\varphi}$ in which the stable focus becomes an unstable one. The loss of stability of the period - 1 cycle gives rise to a stable closed invariant curve, associated with quasiperiodic dynamics.

The asymptotic behavior of the system (7) on the invariant curve depends on the rotation number. If the rotation number is irrational, the invariant curve is densely filled with points of the orbit of map (7), and the dynamics is quasiperiodic. When the rotation number is rational, the closed invariant curve contains a pair of cycles, one of which is stable, while the other is a saddle. The attracting closed invariant curve includes two cycles, a saddle and a stable node, and the saddle-node connection composed of the unstable manifolds of the saddle cycle. Parameters regions corresponding to rational rotation numbers are called Arnold tongues or phase-locking windows $[9,10]$. Figure 2(a) shows also the 1:9 phase-locking window. Here $\alpha_{1}$ and $\alpha_{2}$ are the saddle-node bifurcation points.

The bifurcation behavior of the system (7) is not completely covered by the cases described above. As illustrated in Fig. 2(b) for higher values of the input voltage $U$ more complicated nonlinear phenomena are possible, including multistablilty and hysteretic transitions. The bifurcation diagram in Fig. 2(b) contains three branches denoted as $A, B$ and $C$.

When the parameter $\alpha$ increases, the stable 1 - cycle belonging to the branch $A$ undergoes a Neimark-Sacker bifurcation. In this bifurcation, a closed invariant curve corresponding to the quasiperiodic dynamics softly appears and the 1 -cycle becomes an unstable focus. With further increase in the value $\alpha$, the system enters the region of multistability through a fold bifurcation $[11,12]$.

On the part of this region that falls between the points $\alpha_{2}$ and $\alpha_{3}$ the stable 7 -and 8 -cycles (see branches $B$ and $C$ ) coexist with the quasiperiodic attractor. Here one can observe a hard transition from period -7 cycle to a stable 8 - cycle or quasiperiodic attractor.

\section{Conclusions}

The purpose of this paper was to study some of the complex dynamic phenomena that can arise in a highpower heating unit with pulse width modulated control. The mathematical model of this system was represented as a two-dimensional set of nonautonomous differential equations with discontinuous right-hand sides.

The first step in our investigation was to reduce this system to a two-dimensional piecewise-smooth map (7). We showed, how the high-power heating unit with pulse-width modulated control can exhibit both a transition to quasiperiodic dynamics through a NeimarkSacker bifurcation and a variety of interesting nonlinear phenomena, including phase-locking modes, multistablilty and hysteretic transitions.

In this paper, we discussed only the first steps towards understanding of the observed nonlinear phenomena.

Further development of these investigations is important both from the theoretical and applied points of view, as it will provide a theoretical foundation for design and control of a wide class of pulse-modulated control systems.

The authors would like to thank Prof. Zh.T. Zhusubaliyev for his careful reading of and constructive suggestions to the first draft of this manuscript.

Research is carried out with the financial support of The Ministry of Education and Science of the Russian Federation within the Public contract project 2.1396.2017/PCh.

\section{References}

1. H. J. Scheel, T. Fukuda, Crystal growth technology (John Wiley \& Sons, 2003)

2. H. J. Scheel, P. Capper, Crystal growth technology: From fundamentals and simulation to large-scale production (John Wiley \& Sons, 2008)

3. V.G.Rubanov, A.S. Kizhuk, Y.A. Gol'tsov, Int. J. Soft Comp. 10, (2015)

4. Y.A. Gol'tsov, A.S. Kizhuk, V.G.Rubanov, Int. J. Pharm. Techn. 12, (2016)

5. M.H. Rashid, F.L. Luo, Power Electronics Handbook (Elsevier Science, 2011)

6. Zh.T. Zhusubaliyev, E. Mosekilde, Bifurcations and chaos in piecewise-smooth dynamical system (World Scientific, Singapore, 2003)

7. S.Banerjee, G. C. Verghese, Nonlinear phenomena in power electronics (IEEE Press, New York, 2001)

8. Di. M Bernardo, C. J. Budd, A. R. Champneys, P. Kowalczyk, Piecewise-smooth dynamical systems: Theory and applications (Springer-Verlag, New York, 2008)

9. J.M.T Thompson, H.B. Stewart, Nonlinear dynamics and chaos (Wiley, Chichester, 1986)

10. Yu.A. Kuznetsov, Elements of applied bifurcation theory (Springer-Verlag, New York, 2004)

11. Zh.T. Zhusubaliyev, E. Mosekilde, O.O Yanochkina, IEEE Trans. Power Electron. 26, 4 (2011)

12. Zh.T. Zhusubaliyev, E. Mosekilde, Math. Comput. Simul. 109 (2015) 